\title{
Sidewall-angle dependent mold filling of three-dimensional microcavities in thermal nanoimprint lithography
}

\author{
Helmut Schift, ${ }^{\text {a) }}$ Mirco Altana, ${ }^{\text {b) }}$ and Arne Schleunitz ${ }^{\mathrm{c})}$ \\ Laboratory for Micro- and Nanotechnology, Paul Scherrer Institute, 5232 Villigen PSI, Switzerland
}

(Received 28 June 2012; accepted 11 October 2012; published 26 October 2012)

\begin{abstract}
Nanoimprint stamp cavities with vertical and sloped sidewalls show different filling behaviors during thermal nanoimprinting resulting from the varying ability of the viscous polymer to wet the surface during the thermal imprint step. In this paper, the authors describe the formation of prefill states already in the pressure-less contact phase, before squeeze flow begins to fill the cavities. These stable states are created by a constant contact angle formed at the sidewall, and the states themselves generate surface topographies in the resist below the imprint cavities which are dependent upon the surface energy, the geometry of the stamp cavities, and the resist thickness. Therefore, prefilling topographies, formed once a stamp is in contact with a liquid polymer surface and resulting in a transformation of the resist layer with homogeneous thickness into a thickness modulated film, have to be considered as the standard case in molding. Furthermore, due to the local balance of material pinning and wetting mechanisms, cavities preferentially fill from the sloped sidewall and, therefore, this observed phenomenon is highly relevant for the defect-free replication of 3-D surface topographies. (C) 2012 American Vacuum Society. [http://dx.doi.org/10.1116/1.4764096]
\end{abstract}

\section{INTRODUCTION}

The filling of micro- and nanocavities in thermal nanoimprint lithography (NIL) requires a 3-D flow of viscous material, which means that both the squeeze flow in the lateral direction and filling in the vertical direction have to be induced. ${ }^{1}$ This is valid for cavities with vertical sidewalls (so-called two-dimensional (2-D), binary or two-level structures) as well as cavities with three-dimensional (3-D) profiles (hence called nonbinary). Therefore, in principle the replication of complex surface reliefs is not different from that of binary structures. The ideal case is that the polymer level in the cavity rises homogeneously (i.e., with constant height) until the cavity top is reached due to sinking of the stamp and spatial material displacement which leads to the flow of polymer from the side into the stamp cavities. Finally, the polymer relief assumes a shape conformal to that of the cavity outlines and the complete molding is achieved. However, in addition to squeeze flow which displaces material for filling from below the stamp protrusions to the cavity interior, pinning, wetting, and capillary effects play an important role during molding. ${ }^{2-4}$ In the case of binary structures, this results in spontaneous fast filling of cavities or leads to a complex material redistribution, e.g., capillary bridges, if cavities are extended. While we have found ways to visualize and analyze the squeeze flow and filling of cavities, the formation of these capillary bridges, and particularly their rapid evolution along the sidewalls, is difficult to track. ${ }^{5}$ For nonbinary structures, an even more complex flow is expected; however, until now there was only little knowledge about the mold filling of 3-D cavities because structures

\footnotetext{
a)Electronic mail: helmut.schift@psi.ch

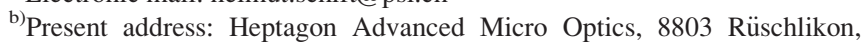
Switzerland.

${ }^{c}$ Present address: Micro Resist Technology GmbH, 12555 Berlin, Germany.
}

with appropriate 3-D sidewalls with a well-defined angle and low surface roughness were not available. ${ }^{6}$ Furthermore, it is difficult to freeze intermediate flow states during the fast wetting and spreading of material along cavity sidewalls and before the onset of squeeze flow. We have now discovered that stable intermediate states are already formed in the pressure-less contact phase between the stamp cavities and the polymer material before squeeze flow sets in. These socalled prefill states are defined by geometry and surface energy rather than by ongoing flow. In this contribution we, therefore, report for the first time on sidewall-dependent filling of nonbinary microcavities in NIL technology resulting from a replication study using stamp structures with vertical and sloped sidewalls and analyze first moldings based on quantitative values of contact-angle-based wetting.

\section{EXPERIMENT}

All stamp structures were fabricated in poly (methyl methacrylate) (PMMA) using modulated-dose electron beam lithography and in the case of nonbinary profiles combined with thermal reflow at moderate temperatures which is described in detail in Refs. 7 and 8. The PMMA pattern was transferred onto silicon by reactive ion etching (RIE) which was subsequently coated with our standard antisticking layer (ASL) using chemical vapor deposition of perfluorinated silanes. ${ }^{9}$ The fabrication of molds using this process gave us more freedom for cavity shapes than standard 3-D manufacturing methods, e.g., V-grooves made by anisotropic etching of Si. For example, this fabrication method allowed the fabrication of ridges where the ends of all angles exhibit vertical sidewalls.

We chose symmetric patterns which resulted in molded line ridges and box-type structures and, in case of inclined sidewalls, truncated pyramids and $\Delta$-ridges with flat tops (and vertical ends for line ridges) were replicated. With a fixed 
height of $1 \mu \mathrm{m}$ and a varying footprint width of 3-5 $\mathrm{m}$, the volume for an individual cavity was kept constant for both nearly vertical $\left(\sim 85^{\circ}\right)$ and sloped $\left(45^{\circ}\right.$ and $\left.30^{\circ}\right)$ sidewall inclinations. In addition to symmetric structures, asymmetric structures with vertical, linearly sloped and bent sidewalls were generated for confirmation of results.

In thermal NIL, a typical imprint process consists of a heating step in a contact regime - a slight pressure is used to ensure contact and thermal equilibration between the stamp, the thin resist layer on the substrate and the heating plates on the top and the bottom of the stack. During this phase, the stack is heated to the imprint temperature, $T_{\text {imprint }}$, above the glass transition temperature, $\mathrm{T}_{\mathrm{g}}$. Because of its relatively high viscosity of around $10^{7} \mathrm{~Pa} \cdot \mathrm{s}$, high pressure (around $10 \mathrm{MPa}$ ) is needed to ensure significant lateral flow. At $T_{\text {imprint }}$, the polymer is kept at imprint pressure until the cavities are filled, and then, the polymer is cooled down below $T_{g}$ to a temperature at which the resist is solidified and the substrate can be easily handled for demolding. For our research, it is essential that the viscous intermediate states can be accessed without any further shape variation during cooling. This is accomplished by interrupting the process in a phase where the polymer has not yet reached the top of the cavities, which enables determining the contact angle (CA) after demolding by scanning electron microscopy (SEM) analysis of resist cross-sections at the cavity sidewalls since the resist contour constitutes an equilibrium state without any further shape variation. In principle, such imprint conditions can be achieved with the fast embossing tool in the Jenoptik HEX03, which is described in detail in Ref. 10. This tool enables one to achieve fast process times with minimum overhead (heating and cooling). As will be shown in Sec. III, most of the results presented here were achieved by standard thermal NIL (i.e., variothermal heating and cooling) with a very low pressure and a rather long imprint time to ensure that the prefilling is finished and stationary. In contrast to Ref. 11, we achieve incomplete filling with this low-pressure regime only and are not aiming at obtaining thin residual layers. A typical imprint of all polymers was performed at temperatures of $40-70^{\circ} \mathrm{C}$ above the glass transition temperatures, $\mathrm{T}_{\mathrm{g}}$, and at a pressure of $0.05 \mathrm{MPa}$ (if not indicated otherwise), with an overall imprint time of $300 \mathrm{~s}$. In all cases, vacuum prior to imprint was needed to avoid air inclusions which could create modification of the $\mathrm{CA}$ formation by the introduction of compressed air.

The imprint polymer used to describe the effect of the sidewall inclination to stamp cavity filling was PMMA with a measured weight averaged molecular weight, $\mathrm{M}_{\mathrm{w}}$, of $34.4 \mathrm{~kg} / \mathrm{mol}$ (denoted as k, PMMA-25k from Polysciences, Inc.). To ensure the accuracy of the CA measurements performed at the prefilled structures, additional PMMA polymers were chosen (with a measured $\mathrm{M}_{\mathrm{w}}$ of $91.2 \mathrm{k}$ and $8.9 \mathrm{k}$ ).

Since we are most interested in cases where the polymer surface stays in a state between the pinning (at the bottom of the sidewall) and the wetting of the cavity top (i.e., at the top of the sidewall), SEM cross-sections far from the end of polymer ridges were accomplished by adequate sample cleavage. From these cross-section profiles, the CA was derived within an accuracy of about $\pm 1^{\circ}$ (within the accuracy of the CA measurement equipment). The actual tolerance has to be set slightly higher (about $\pm 2^{\circ}$ ) due to the fact that cleaved samples are taken and that both the sidewall inclinations and the CA have to be determined via optical means.

\section{RESULTS AND DISCUSSION}

\section{A. Results}

Stable intermediate states for both binary and nonbinary structures, as shown in Fig. 1, were achieved using the lowpressure embossing approach. For this, arrays of square cavities with a footprint width of 3 and $4 \mu \mathrm{m}$ and vertical and inclined sidewalls were imprinted. As expected, the cavities are continuously filled by squeeze flow, starting from the array boundary to the center. Furthermore, an increase in pressure results in faster filling and reveals differences in the binary and nonbinary cases. In the case of inclined sidewalls, however, we even see partial filling in the center, though squeeze flow has not reached the related cavities. Moreover, it seems that all the incompletely filled cavities exhibit a similar shape, assuming that it is determined by the sidewall geometry. Figure 2 shows that for vertical sidewalls of small box-type structures, this filling happens along a few periods only, with an almost abrupt start of the filling at the corners of the individual cavities. The filling of such cavities seems to be entirely driven by the capillary effect and does not continue if pressure does not enable flow from the side. Due to the fact that we wanted to investigate prefilling without superposition of squeeze flow, i.e., a stable state which forms at negligible pressure only due to the wetting of the polymer, we therefore considered the low pressure imprint regime as appropriate for our research.

In order to further analyze the observed prefilling states, long extended microcavities were investigated which allowed

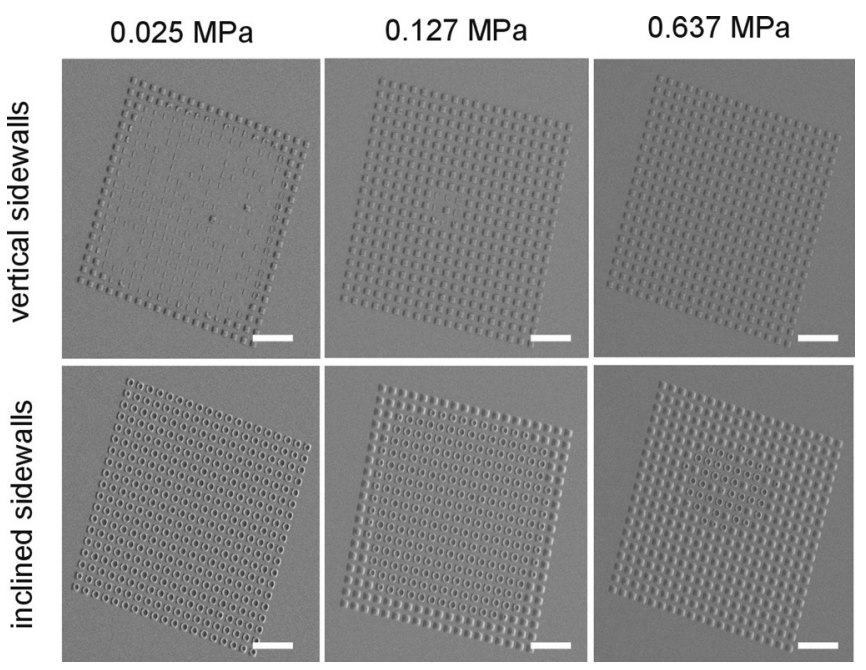

FIG. 1. SEM-micrographs of the filling of arrays with squares with vertical and inclined sidewalls (scale bar $20 \mu \mathrm{m}$ ). Squeeze flow leads to gradual filling of patterns starting from the array boundary to the center, depending on the pressure. While for vertical sidewall cavities, unfilled cavities are found in the center, for inclined sidewalls, the cavities are partially filled due to capillary action (called prefilled). 

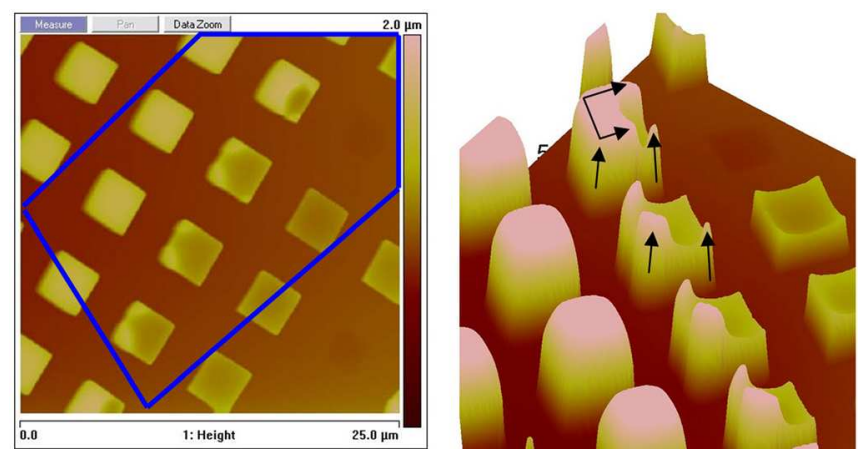

FIG. 2. (Color online) AFM-micrograph (2D and 3D representation) of molded $4 \times 4 \mu \mathrm{m}^{2}$ box-type structures at the transition from an unfilled (upper right side) to a completely filled state (left). In the intermediate state during filling (center), as the black arrows indicate, the filling occurs at the corners and the top surface of the cavities. Additionally, prefilled states (right) can be distinguished with a defined meniscus.

easy acquisition of cross-sectional views simply by cleaving (see Fig. 3). Here, it was found that capillary forces can delocalize considerable amounts of polymer over large distances, as was already observed along extended ridges. ${ }^{12}$ Induced by the upward movement of the polymer into the cavities resulting from capillary forces, a considerable amount of polymer is pulled from the inside of the cavity leading to local material depletion beneath the stamp cavity. We therefore expect these prefill states to be even more pronounced for more complex profiles and, consequently, the dominant force during mold filling of specific structures. Cross-sections need to be taken in areas where these prefill states represent the lateral flow into the cavities. Ridge cavities with vertical sidewalls seem to fill from the end, being able to pull material from inside the ridge, resulting in a large delocalization of material along the ridge. In contrast, ridges with inclined sidewalls seem to fill more homogeneously along the ridge assuming that the pinning at the cavity borders is less severe. Due to volume conservation, material has to flow from inside the cavity to the borders, leading to local depletion and defects which might be difficult to smooth out during filling.

For all sidewall inclinations, the CA between the stamp surface and the imprinted polymer was determined. In the case of PMMA $\mathrm{M}_{\mathrm{w}} 34.4 \mathrm{k}$ this was found to be $90^{\circ}$. While a CA of $90^{\circ}$ means that an almost horizontal surface level forms between two neighboring vertical cavity sidewalls, for symmetric structures with inclined sidewalls a lens-like depletion (i.e., a full meniscus) of polymer below the cavity center is formed by the surface minimization effect. In Fig. 4, two different CAs were determined from an imprint of $30^{\circ}$ slopes in mr-I $8150 \mathrm{E}$ XP and in PMMA $\mathrm{M}_{\mathrm{w}} 8.9 \mathrm{k}$ to be $98^{\circ}$ and $85^{\circ}$, respectively. The employed resist thickness
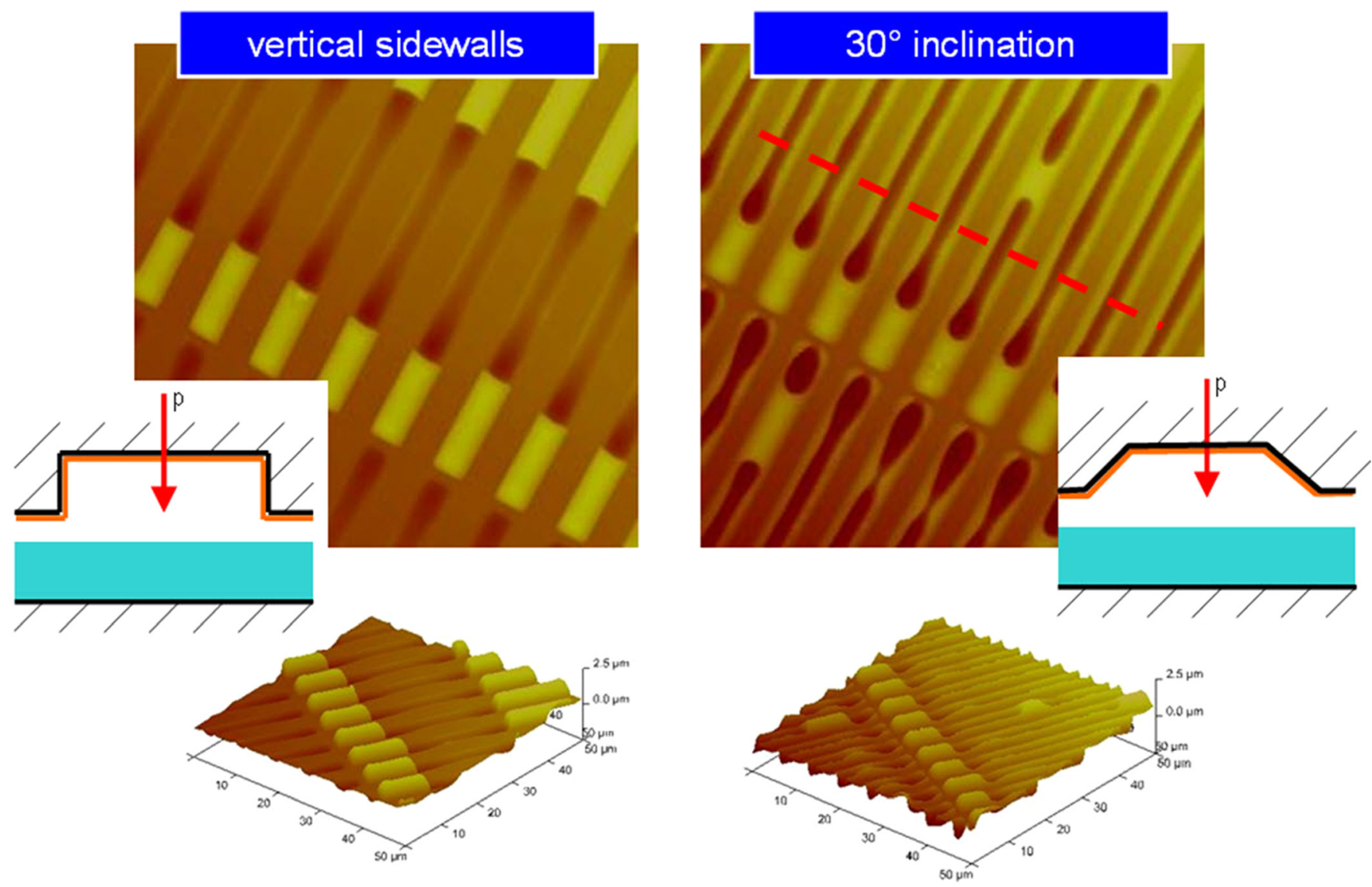

FIG. 3. (Color online) AFM micrographs (2D and 3D representation) of the filling study showing prefill states with a line ridge with vertical and inclined sidewalls at the end for identical imprint conditions. Both ridges fill at the ends due to vertical end-sidewalls. While this is the dominant factor for vertical sidewalls, sloped sidewalls enable surface wetting and filling along the sidewalls, too. In both cases, stable prefill cases can be found and, therefore, cross-sections in the center of the ridge enable a study of the wetting of the sidewalls. 


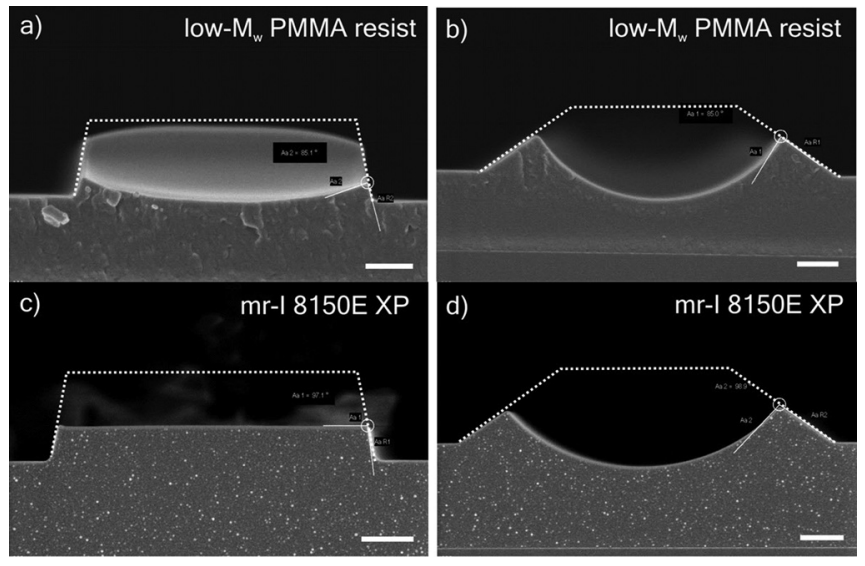

FIG. 4. SEM migrographs demonstrating the formation of prefilling states for vertical $\left(\sim 85^{\circ}\right)$ and inclined sidewalls $\left(30^{\circ}\right)$ in imprinted resist $(1000 \mathrm{~nm}$ thick), exemplified (a), (b) for PMMA $\mathrm{M}_{\mathrm{w}} 8.9 \mathrm{k}$ with $85^{\circ}$, and for (c), (d) $\mathrm{mr}-\mathrm{I} 8150 \mathrm{E}$ XP with a contact angle of $98^{\circ}$ (scale bar $1 \mu \mathrm{m}$ ).

of $1 \mu \mathrm{m}$ is in the range of the cavity height. Note that for vertical sidewalls (or in case of the nearly vertical sidewalls at $85^{\circ}$ ) some flow of material into the cavity is needed to achieve a level above the initial resist thickness, which also means that the pinning at the lower corner of the cavity has to be overcome (resulting in a change in flow direction of $90^{\circ}$ ). In contrast to this rather abrupt behavior, in the inclined case the polymer tends to spread easily across the less sharp corners to wet the sloped surfaces, leading to a constant rise of polymer material along extended sidewalls which stops when the energetic favorable surface shape is reached.

As already mentioned, for different sidewall inclinations the CA remains identical, which was confirmed for $30^{\circ}$ and $45^{\circ}$ and for vertical sidewalls. The shape of a lens- or cylinder-like depression is defined by the CA of the polymer with respect to the sidewall, the size and sidewall inclination of the cavity, and also by the resist thickness (see Fig. 5). In the case of wide cavities (e.g., $30^{\circ}$ inclination and $90^{\circ} \mathrm{CA}$ in a $5 \mu \mathrm{m}$ wide cavity), this can lead to a depletion $0.5 \mu \mathrm{m}$ below the initial level of the resist; therefore, a full meniscus could reach down to the substrate. If the resist is thinner $(\sim 0.5 \mu \mathrm{m})$, the CA still remains the same but the meniscus formation is suppressed. That is why the prefilling exhibits lower heights in cases with 300 and $150 \mathrm{~nm}$ resist thickness: a thin layer of polymer always remained on the substrate and thus dewetting was not observed.
TABLE I. Correlation of measured contact angles of molten polymer beads of PMMA $\mathrm{M}_{\mathrm{w}} 34.4 \mathrm{k}$ for different temperatures and comparison with prefilling in a cavity with a $30^{\circ}$ inclination.

\begin{tabular}{lllll}
\hline \hline PMMA & Reflow & Reflow & Reflow & Prefilling \\
$34.4 \mathrm{k}$ & $160^{\circ} \mathrm{C}$ & $180^{\circ} \mathrm{C}$ & $200^{\circ} \mathrm{C}$ & $\sim 190^{\circ} \mathrm{C}$ \\
$\mathrm{CA}$ & $96^{\circ}$ & $91^{\circ}$ & $85^{\circ}$ & $90^{\circ}$ \\
\hline \hline
\end{tabular}

In order to prove the accuracy of the CA measurements using SEM, prefill states of different polymers were generated and analyzed. In every case, the CAs in cavities with different sidewall inclinations varied only to a small extent for each polymer (see Fig. 4).

The CA measurements by SEM inspection were proved applicable also by reflowing macroscopic PMMA beads at different temperatures. ${ }^{13}$ In this experiment, beads of granulate size around $300 \mu \mathrm{m}$ were put on a silicon surface and subsequently heated on a hot plate to a specific temperature for $20 \mathrm{~h}$. The long time was chosen for comparison with results achieved in Ref. 12, but for ASL-coated substrates do not deviate from those obtained at much shorter time scales. It is well known that the surface energy and, therefore, the $\mathrm{CA}$ is temperature dependent; ${ }^{6}$ consequently, we obtained CAs of PMMA beads at different temperatures. As can be seen in Table I for PMMA $34.4 \mathrm{k}$, the CA of the prefill at a $190{ }^{\circ} \mathrm{C}$ imprint temperature is comparable to that of reflown beads between 180 and $200^{\circ} \mathrm{C}$.

Finally, asymmetric structures with different cavity widths were imprinted (see Fig. 6). As before, due to the inclination of one sidewall a meniscus was formed. Due to the pinning on the lower corner of the vertical sidewall, different widths resulted in different heights of filling at the inclined sidewall. For asymmetric lens-like structures with a gradually declining slope angle at one side and a vertical sidewall at the other (as in Fig. 7), it can be observed that the CA remains constant up to the top of the cavity and, thus, is independent of the prefilling height. Furthermore, a preferential filling from one side can be achieved.

\section{B. Discussion}

The main finding of this research is that early in the contact regime of an imprint process, i.e., before the build-up of the full imprint pressure, capillary action can pull viscous resist material along cavity sidewalls into mold cavities while other areas are depleted of polymer. This means that
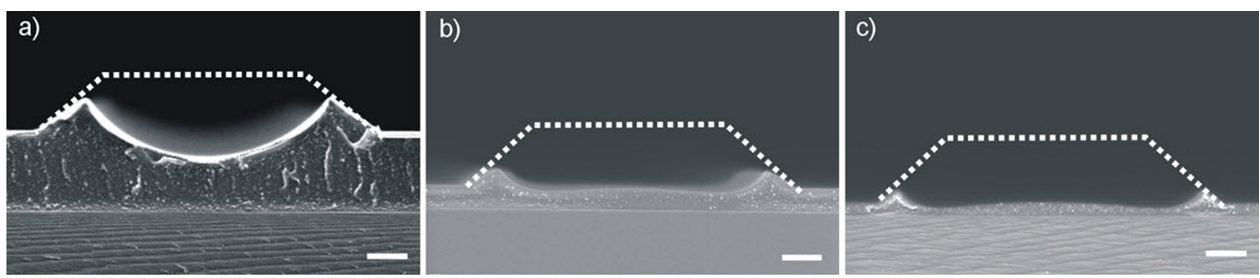

FIG. 5. (Color online) SEM micrographs of imprints of a symmetric stamp structure with inclined sidewalls $\left(30^{\circ} \mathrm{C}\right.$ on both sides of the ridge-like stamp cavity) for different resist thicknesses. A lens- or cylinder-like depression below the cavity can only form for thick resist layers, assuming the optimum contact angle. For thin resists, the same contact angle forms at the sidewalls but, instead of depletion down to the substrate, flow toward the borders is inhibited and the substrate stays wetted (scale bar $1 \mu \mathrm{m})$. 

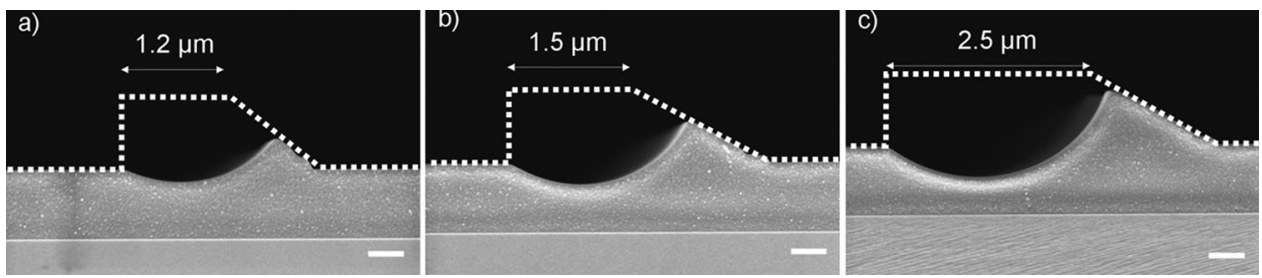

FIG. 6. SEM micrographs of imprints of asymmetric stamp structures with one vertical and one inclined sidewall and different cavity widths (scale bar $1 \mu$ m). Due to the pinning at the bottom of the vertical sidewall and the constant contact angle at the inclined sidewall, the depth of the meniscus increases for wider cavities.

within certain areas of the cavities part of the polymer is pulled above its initial level which, due to volume conservation, leads to a lowering of the resist level in other areas. Once they have been generated these states are stable and, thus, can be visualized and analyzed by cooling the imprinted polymer below $\mathrm{T}_{\mathrm{g}}$. Without the presence of squeeze flow, the maximum prefilling height is determined by the sidewall inclination, the contact angle, and geometrical factors such as cavity width and resist height. For CAs of around $90^{\circ}$, this means that for vertical sidewalls no filling occurs, and for inclined sidewalls, a depression below the original polymer level forms at specific initial resist thicknesses. While CAs around $90^{\circ}$ on ASL-coated surfaces seem to apply for a range of polymers, such as cyclic olefin copolymer ${ }^{13}$ and polystyrene,${ }^{14}$ it is obvious that for smaller CAs, as would also be the case for surfaces without ASL, depressions will also form in the case of vertical sidewalls. Contact angles higher than $90^{\circ}$, in contrast, will lead to mounds similar to those formed by plug flow. ${ }^{14}$

When squeeze flow sets in, more material will flow into the cavity and the stamp will sink. This leads to a rise of the polymer level in the cavity but, as has been shown in Fig. 3, movement in the longitudinal direction of the ridges also pulls this additional material toward the ends. Particularly in the case of vertical sidewalls, where pinning at the cavity corner has to be anticipated, this longitudinal flow might be energetically more favorable than lateral flow. In general, the dynamics is complex and competition takes place between forces favoring pinning and lateral and longitudinal flow. This is also a first indication that in the case of vertical sidewalls, the filling does not start at a preferential spot within a large cavity but rather facilitates the forming of capillary bridges to the cavity top due to slight resist surface undulations. ${ }^{2,3}$ In addition, the polymer starts easily at corners where two vertical sidewalls meet, leading to a fast pulling of polymer from the cavity center to these corners. This is a possible explanation why, for structures with inclined sidewalls and for polymers with a CA of less than $90^{\circ}$, a formation of capillary bridges was not observed.

\section{SUMMARY AND CONCLUSIONS}

Until now the effect of prefilling states for molding of micro- and nanostructures in thermal NIL was barely described, since it was very difficult to visualize these transient and stable-flow states in the microregime during fast wetting and spreading at the onset of flow. In the research presented here, we report on stable prefilling states observable in a low-pressure regime, i.e., the contact force regime during heating in typical thermal imprint processes. These studies show that prefilling is not only an effect dependent upon the surface energies of polymers and stamps but, more importantly, upon cavity geometry and sidewall inclinations. Thus, sidewall geometry has a great influence on how cavity filling is initiated and which kind of surface topographies are forming before squeeze flow sets in. In particular, depleted areas can act as hot spots which may be difficult to erase once the depression has formed and, therefore, may lead to defects. In contrast, other spots, like corners in square cavities with vertical sidewalls, may be filled faster than predicted by current squeeze flow simulations. Moreover, it is possible that during incomplete molding, the formation of prefilling states was often unknowingly misinterpreted in the past as trapped air or demolding defects.

For typical variothermal imprint processes with an extended contact regime (a few tens of seconds for temperature equilibration before the full pressure is applied), we can now assume that prefill states distributed over the entire resist surface are present before the squeeze flow starts. This is particularly important for future simulations which would
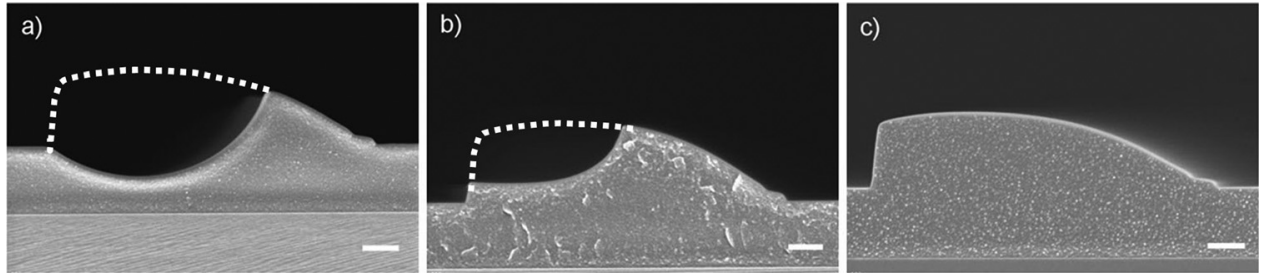

FIG. 7. SEM micrographs of imprints of asymmetric stamp structures with one vertical and one gradually inclined sidewall with different cavity widths (scale bar $1 \mu \mathrm{m}$ ), beginning at $30^{\circ}$ and reaching up to the horizontal lens-like top of the cavity. Due to the tendency to keep a constant contact angle at the inclined sidewall up to the top of the cavity during slight filling with squeeze flow, the pinning at the bottom of the vertical sidewall (a) is overcome and the polymer begins to rise (b). In (c), a completely filled cavity is shown, which also represents the shape of the asymmetric cavity. 
need to predict this filling not starting from spincoated resist surfaces with homogeneous thickness but from prepatterned films with defined surface corrugations or even voids and areas of depletion which might lead to "starvation" of resist flow. Therefore, we no longer can consider starting an imprint from a resist layer of homogeneous thickness from which squeeze flow fills the cavities. Instead, we have to take the prefilled states into account with thickness variations from specific shapes (depressions and mounds). However, since we do not yet know the speed of prefill state formation, fast (nonvariothermal) processes with minimized contact time may circumvent this. A further result is that prefilling is dependent on the surface energy of polymers, especially for liquid UV-NIL resins where capillary filling even dominates squeeze flow in low-pressure regimes. For highspeed molding, it is of great importance to find optimum combinations of substrate, mold, and polymer surface energies to achieve fast and defect-free molding.

\section{ACKNOWLEDGMENTS}

The authors thank K. Vogelsang and C. Spreu (PSI, Villigen), as well as M. Vogler (Micro Resist Technology $\mathrm{GmbH}$, Berlin) and M. Roux (FHNW, Windisch) for their help and valuable contributions. The research presented here was partially funded by the Swiss Federal Office for Science and Education in the framework of the EC-funded project NaPANIL (Contract No. NMP 214249). The content of this work is the sole responsibility of the authors.

${ }^{1}$ L. J. Heyderman, H. Schift, C. David, J. Gobrecht, and T. Schweizer, Microelectron. Eng. 54, 229 (2000).

${ }^{2}$ S. Y. Chou and L. Zhuang, J. Vac. Sci. Technol. B 17, 3197 (1999).

${ }^{3}$ H. Schift, L. J. Heyderman, M. Auf der Maur, and J. Gobrecht, Nanotechnology 12, 173 (2001).

${ }^{4}$ S. Landis, N. Chaix, D. Hermelin, T. Leveder, and C. Gourgon, Microelectron. Eng. 84, 940 (2007).

${ }^{5}$ H. Schift, G. Kim, J. J. Lee, and J. Gobrecht, Nanotechnology 20, 355301 (2009).

${ }^{6}$ N. Bogdanski, S. Mollenbeck, and H.-C. Scheer, J. Vac. Sci. Technol. B 26, 2416 (2008).

${ }^{7}$ A. Schleunitz and H. Schift, J. Micromech. Microeng. 20, 095002 (2010).

${ }^{8}$ A. Schleunitz and H. Schift, Microelectron. Eng. 88, 2736 (2011).

${ }^{9}$ H. Schift, S. Saxer, S. Park, C. Padeste, U. Pieles, and J. Gobrecht, Nanotechnology 16, S171 (2005).

${ }^{10}$ H. Schift, S. Bellini, M. B. Mikkelsen, and J. Gobrecht, J. Vac. Sci. Technol. B 25, 2312 (2007).

${ }^{11}$ N. Bogdanski, M. Wissen, S. Mollenbeck, and H.-C. Scheer, J. Vac. Sci. Technol. B 24, 2998 (2006).

${ }^{12}$ H. Schift, C. Spreu, A. Schleunitz, and J. J. Lee, Microelectron. Eng. 88, 87 (2011).

${ }^{13} \mathrm{M}$. Matschuk, "All-polymer microfluidic system with integrated nanostructures for cell handling," Ph.D. dissertation (Technical University of Denmark (DTU), 2011).

${ }^{14}$ A. Mayer, S. Mollenbeck, and H.-C. Scheer, "Contact angles in a thermal imprint process," Abstract Proceedings and Oral Presentation at EIPBN Conference, Waikoloa, HI, 29 May-1 June 2012. 